\title{
Turbulence simulation in diverse conditions for FSO Links
}

Heba Yuksel Hasim Meric 


\title{
Turbulence simulation in diverse conditions for FSO Links
}

\author{
HEBA YUKSEL ${ }^{1}$, HASIM MERIC ${ }^{2}$ \\ ${ }^{1}$ Bogazici University, Dept. of Electrical-Electronics Eng. Istanbul, Turkey \\ e-mail: heba.yuksel@boun.edu.tr \\ ${ }^{2}$ Bilkent University, Dept. of Electrical-Electronics Eng. Ankara, Turkey \\ e-mail: hasim@bilkent.edu.tr
}

\begin{abstract}
Simulation of beam propagation through turbulent media has always been a tricky subject when it comes to moderate-to-strong turbulent regimes. Creating a well controlled turbulent environment is beneficial as a fast and a practical approach when it comes to testing the optical wireless communication systems in diverse atmospheric conditions.

Turbulent media is created using multiple phase screens each having controlled random variations in its frequency and power while the propagated beam is calculated using Fresnel diffraction method. The effect of the turbulent media is added to the propagated beam using modified Von Karman spectrum. Created scintillation screens are tested and compared with the experimental data which are gathered in different turbulence regimes within various atmospheric conditions. We believe that the general drawback of the beam propagation simulation is the difference in terms of spatial distribution and sequential phase textures. To overcome these two challenges we calculate the Aperture Averaging Factors to create more realistic results. In this manner, it is possible create more viable turbulent like scintillations thus the relationship between the turbulence strength and the simulated turbulence parameters are distinctly available.
\end{abstract}

Our simulation gives us an elusive insight on the real atmospheric turbulent media. It improves our understanding on parameters that are involved in real time intensity fluctuations that occur in every wireless optical communication system.

Keywords: Simulation of Turbulence, Free Space Optical Communication, Optical Wireless Link, Strong Turbulence, Scintillation Index $\mathrm{C}_{\mathrm{n}}{ }^{2}$, Aperture Averaging, Phase Screen Method.

\section{INTRODUCTION}

Experimenting on a free space optical communication system is rather tedious and difficult. The interferences of plentiful elements are always affecting the result and causing the experimental outcomes to have bigger error variance margins than they are supposed to have. Especially when we go into the stronger turbulence regimes the simulation and analysis of the turbulence induced beams requires delicate attention [1].

For all these purposes a method for creating turbulence effects on a propagated beam in a desired experiment conditions for all turbulence regimes is presented in this paper.

We have used a point source which combines the Gaussian and sinc forms in 2D [2, 3],

$$
p t_{\text {gaus }-\sin c}=e^{\frac{-j k}{2 D_{z}} r^{\wedge 2}} \frac{D_{o b}}{\lambda D_{z}} \sin c\left(\frac{D_{o b}}{\lambda D_{z}} x_{1}\right) \sin c\left(\frac{D_{o b}}{\lambda D_{z}} y_{1}\right)
$$

where $D_{z}$ is the propagation distance, $D_{o b}$ is the diameter of the observation aperture, $\mathrm{r}$ is the spatial polar coordinate and the $\mathrm{k}$ is the wave number.

Laser Communication and Propagation through the Atmosphere and Oceans, edited by Alexander M. J. van Eijk, Christopher C. Davis, Stephen M. Hammel, Arun K. Majumdar, Proc. of SPIE Vol. 8517, 85170H - (C) 2012 SPIE · CCC code: 0277-786X/12/\$18 - doi: 10.1117/12.929592 
In the act of propagation through air, a super-Gaussian absorbing boundary is applied spatially through for addition of random phase screens to the propagated beam [4],

$$
G_{S u p G}=e^{-\frac{1}{2}\left(\frac{r}{\sigma}\right)^{8}}
$$

where $\mathrm{r}$ is the location parameter of polar coordinates and $\sigma$ is about the half of the number of grids $(\mathrm{N})$.

For the propagation of the beam, angular spectrum form of Fresnel diffraction has been used [2],

$$
U\left(r_{2}\right)=F^{-1}\left[r_{2}, f_{1}\right] H\left(f_{1}\right) F\left(f_{1}, r_{1}\right)\left\{U\left(r_{1}\right)\right\}
$$

where $H(f)$ is the transfer function of the free space propagation given as,

$$
H\left(f_{1}\right)=e^{j k \Delta z} e^{-j \pi \lambda \Delta z\left(f_{x 1}^{2}+f_{y 1}^{2}\right)}
$$

Von Karman spectrum has been chosen for its identity with Kolmogorov spectrum when $\mathrm{l}_{0}=0$ and $\mathrm{L}_{0}=\infty$,

$$
P S D_{\text {mod_VON }}=0.033 C_{n}^{2} \exp \left(-\frac{\left(\kappa / \kappa_{m}\right)^{2}}{\left(\kappa^{2}+\kappa_{0}^{2}\right)^{11 / 6}}\right)
$$

where, $\kappa_{m}=\frac{5.92}{l_{0}}$ and $\kappa_{0}=\frac{2 \pi}{L_{0}}$

\section{MATERIALS AND METHODS}

\section{Methodology of Turbulence creation:}

Simulation of beam propagation in a turbulent media is explained in many literatures $[2,3,56$,]. In the preceding section we are offering a methodology for creating real-time turbulence like atmospheric effects on a propagated beam.

Using the point source in the form of Eq. 1, we have established a propagation pattern at a distance $\mathrm{D}_{\mathrm{z}}$. After defining equidistance observation plane locations between the source plane and the final observation plane, the same numbers of turbulence phase screens have been created to influence the propagated beam. The methodology for the turbulence phase screen is described in the following paragraphs.

Fast fluctuations, which are found to be the effective parameter that ies the strong turbulence, are applied to the propagated beam for each screen defined by Fresnel diffraction method where $\Delta_{o b}$ is the observation plane gird spacing and the N is the FFT factorization number.

Depending on the observation grid spacing, the frequency grid is also established,

$$
f_{\text {fast }}=(-N / 2: N / 2-1) \times \frac{1}{N \times \Delta_{o b}}
$$

Using Eq. 5, we have calculated the associated power that is induced by the fast fluctuations where $\kappa=\frac{2 \pi}{\lambda_{\text {fast }}}$ and $\lambda_{\text {fast }}=\frac{c}{f_{\text {fast }}}$. 
In the preceding sections, the calculation of power spectrum for both lower and faster fluctuations, other than applying a constant $\mathrm{C}_{\mathrm{n}}{ }^{2}$ we are offering a new method. For Turbulence regimes that are moderate to strong $\left(\sigma_{R}>1\right)$, we believe that variations of the power fluctuations that are induced to the propagated beam gets bigger in the temporal region. So in the process of calculation of each turbulence phase screen, the $\mathrm{C}_{\mathrm{n}}^{2}$ variation increases even after short distances. Also by taking the effect of angular spectrum form of Fresnel diffraction into account (Eq. 3.), introducing an error margin on the power that is induced to the propagated beam in each turbulence phase screen will be effective in reaching the desired turbulence strength.

The $\mathrm{C}_{\mathrm{n}}{ }^{2}$ function for strong turbulence regimes that is used in this work is given as,

$$
C_{n_{-} \text {PhsScrn }}^{2}=\operatorname{normrnd}\left(C_{n}^{2}, C_{n}^{2} \times \frac{\sigma_{R}^{2}}{100}\right)
$$

which generates us a normal distributed number with $C_{n}^{2}$ mean and a variance of $C_{n}^{2} \times \frac{\sigma_{R}^{2}}{100}$.

Then normal distributed random numbers with a unity power of $(\mathrm{NxN})$ size phase screen is created. Power associated for each pixel point is assigned to the appropriate planar frequency as a Fourier coefficient of the phase screen,

$$
c_{n f a s t}=(\operatorname{rand}(N x N)) \times \sqrt{P S D_{\text {fast }}} \times \Delta_{o b}
$$

Inverse Fourier transform is taken in 2D for realizing the fast fluctuation effect of the turbulence phase screen $\Phi_{\text {high }}$,

$$
\Phi_{\text {high }}=F\left\{c_{\text {nfast }}\right\}^{-1}
$$

Resulted fast fluctuations can be seen in Figure 1,

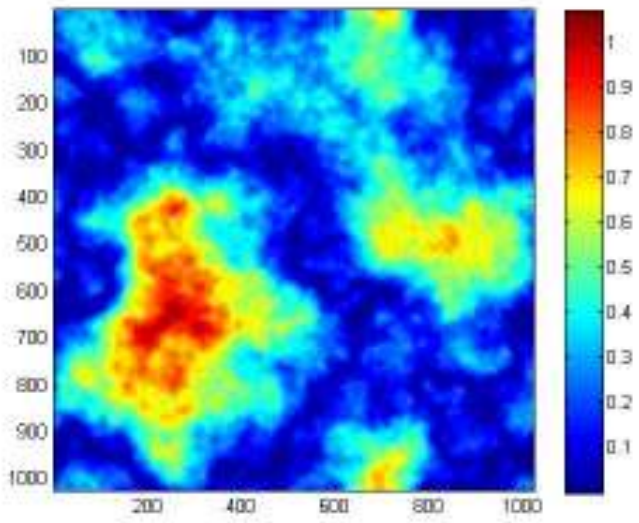

Fig. 1. Fast Fluctuation Effect of the Optical turbulence on a 100 meter slant path with a $\mathrm{C}_{\mathrm{n}}{ }^{2}=10^{-16}$.

Lower fluctuations have been accomplished using the same rule applying a larger frequency grid,

$$
f_{\text {low }}=[-1,0,1] \times \frac{1}{3^{\text {Round }\left(3.4 /\left(C_{n}^{2} \times 10^{16}\right)\right)} \times N \times \Delta_{o b}}
$$


Again using Eq. 5, we have calculated the associated power that is induced by the lower fluctuations where $\kappa=\frac{2 \pi}{\lambda_{\text {low }}}, \lambda_{\text {fast }}=\frac{c}{f_{\text {low }}}$

Then the Fourier coefficients associated with the lower fluctuations are calculated by,

$$
c_{\text {nlow }}=(\operatorname{rand}(N x N)) \times \sqrt{P S D_{\text {low }}} \times \Delta_{o b}
$$

And the turbulence phase screen for lower fluctuations is found by taking an inverse Fourier transform,

$$
\Phi_{\text {low }}=F\left\{c_{\text {nlow }}\right\}^{-1}
$$

Resulted fast fluctuations can be seen in Figure 2,

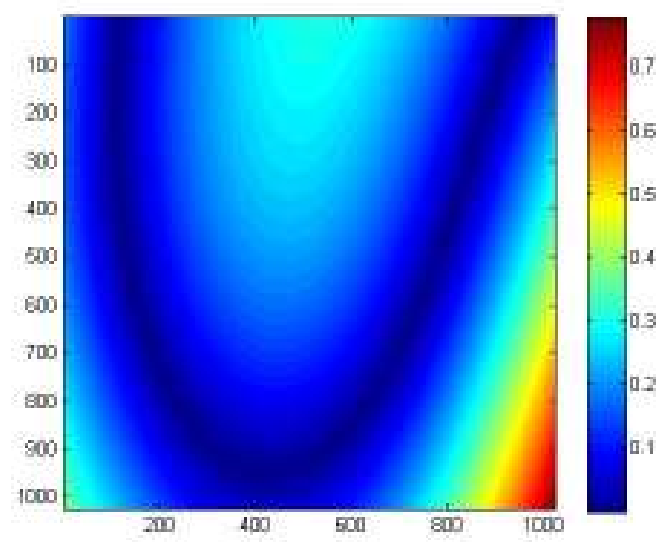

Fig. 2.Lower Fluctuation Effect of the Optical turbulence on a 100 meter slant path with a $C_{n}{ }^{2}=10^{-16}$.

Addition of two effects have created a practical turbulence screen with pre-known turbulence structure parameter $\left(\mathrm{C}_{\mathrm{n}}{ }^{2}\right)$,

$$
\Phi_{\text {TOT }}=\Phi_{\text {fast }} \times e^{\sigma_{R}-1}+\Phi_{\text {low }}
$$

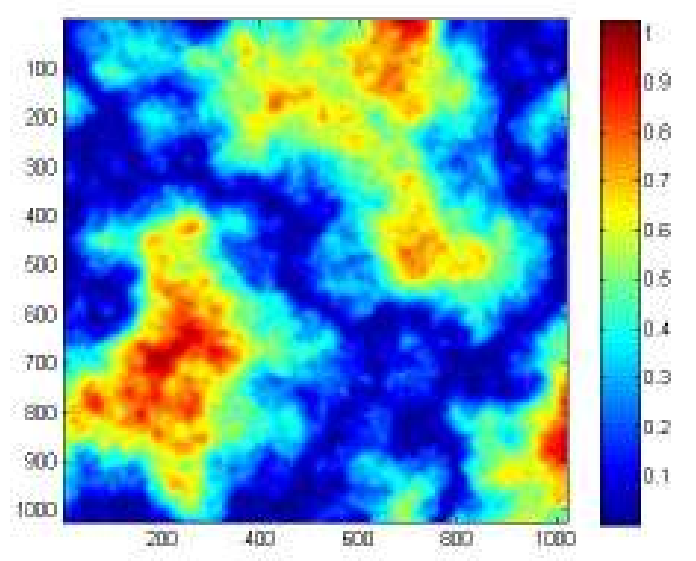

Fig. 3.Simulation Result of Optical turbulence screen on a 100 meter slant path with a $C_{n}{ }^{2}=10^{-16}$.

Resulted optical turbulence screen have been collimated with the each sequential observation plane. The influence of the turbulence phase screen has been passed through while we propagate using Eq. 3 until the last observation plane. 


\section{RESULTS}

As a result, we have a perfect scintillation pattern which has a specific turbulence strength structure constant $\left(\mathrm{C}_{\mathrm{n}}^{2}\right)$ with a predefined propagation distance. In Figures 4-6, we can see the observation plane of a $633 \mathrm{~nm}$ beam after 100 meters of distance in various turbulence strengths.
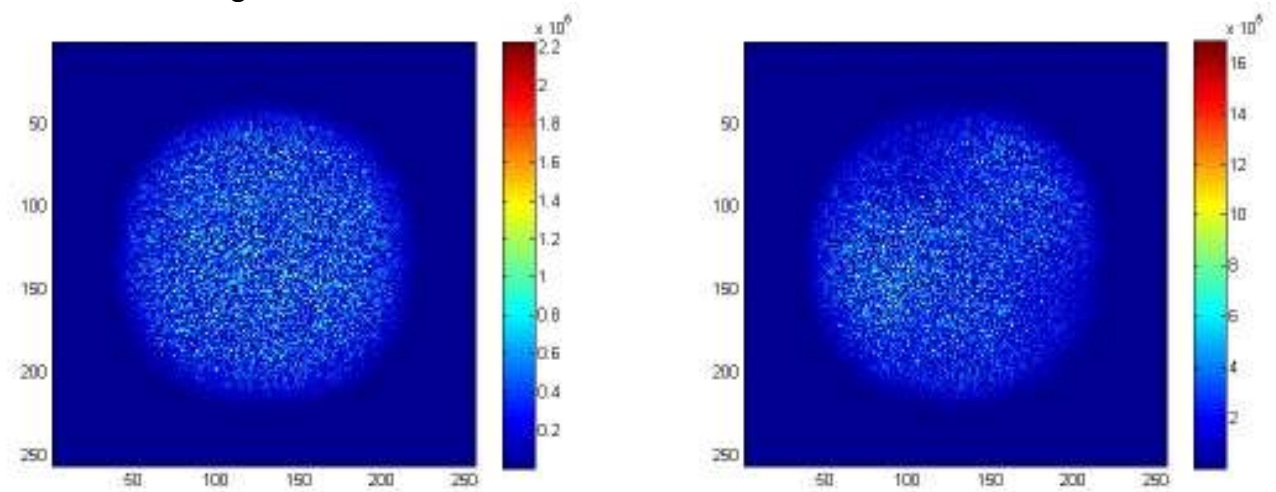

Fig. 4. Strong Turb. Image, $\mathrm{Cn} 2=10^{-10}$, Path:100 m. Fig. 5. Intermediate Turb. Image, Cn2 $=10^{-13}$, Path: $100 \mathrm{~m}$..

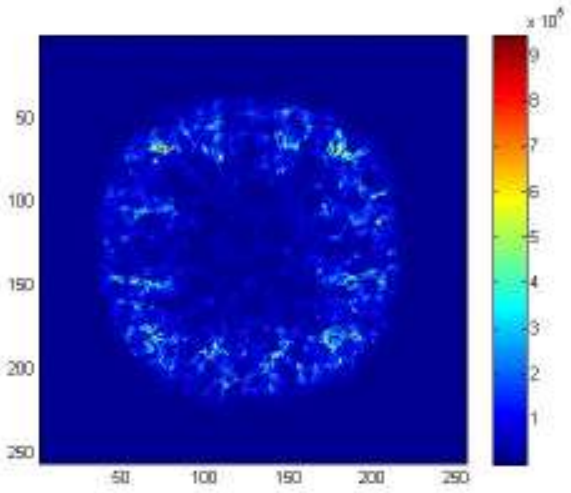

Fig. 6. Weak Turb. Image, $\mathrm{Cn} 2=10^{-15}$,'Path:100 m.

The simulation results of the Aperture Averaging factors for varying Aperture Sizes in various Turbulence regimes is given in Figure 7.

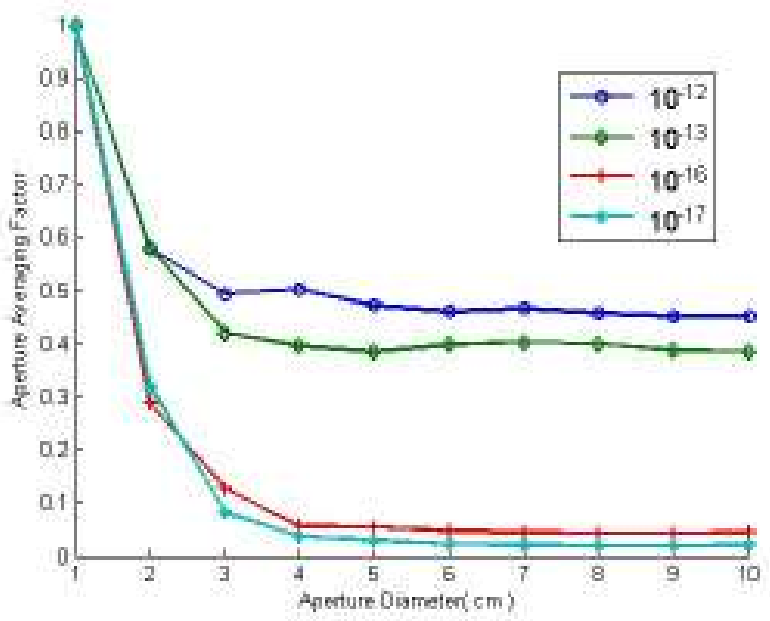

Fig. 7. Simulated Aperture Averaging Results over 100 meters for various $\mathrm{C}_{\mathrm{n}}{ }^{2}$ values 
AA Factor for the strong turbulence regime is given in Figure 8. Experimental results in the strong turbulent regime shows us that the declination of the curve is very similar to our simulation results. There is a \%10 error margin in the saturation region of the Aperture Averaging curve which is acceptable in strong turbulent regimes.

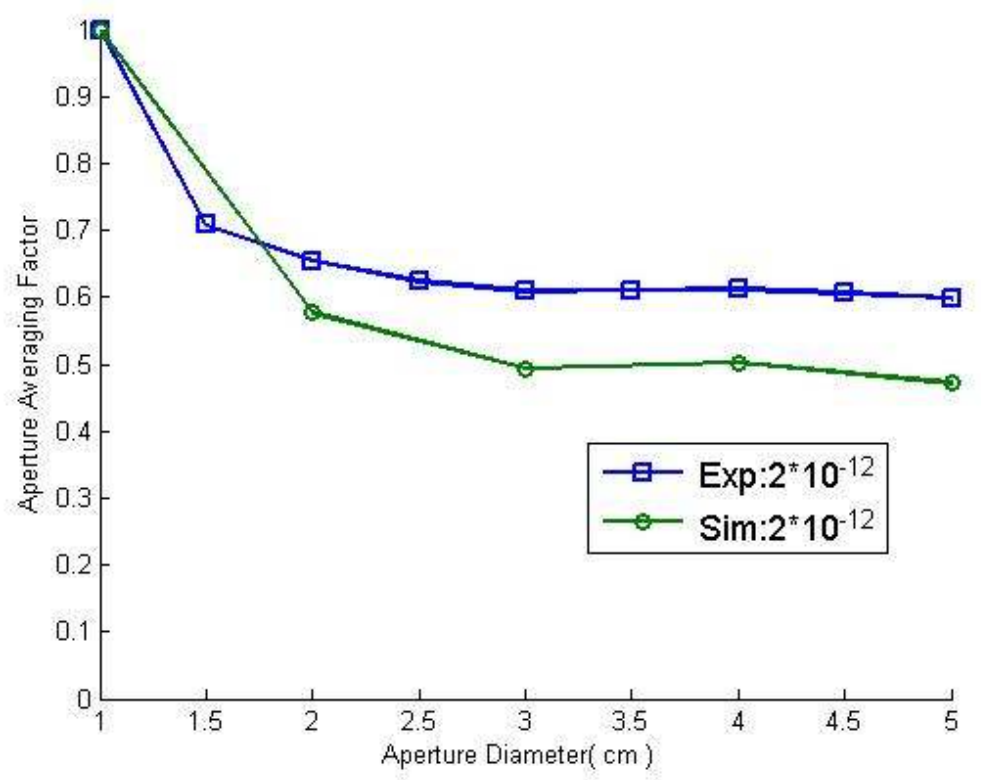

Fig. 8. Experimental and Simulated Results of Point Averaged Aperture Averaging Factor where $\mathrm{C}_{\mathrm{n}}{ }^{2}=2 \times 10^{-12}$ and path length $=120$ meters.

AA Factor for weak-to-moderate turbulence regime is given in Figure 9. Experimental results seem to match up with the simulation results in the saturation regime of the curve. It is our idea that the declination difference is caused by number of phase screens that are in use. The difference can be decreased by increasing the number of sampling we take from the observation plane for each realization.

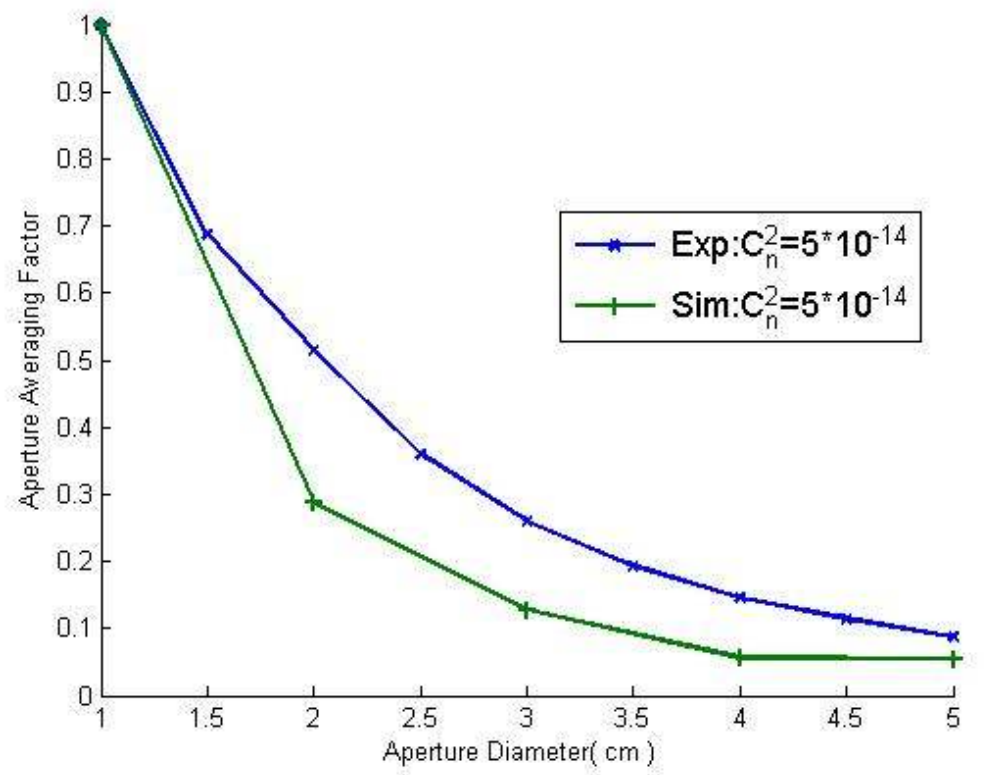

Fig. 9. Experimental and Simulated Results of Point Averaged Aperture Averaging Factor where $\mathrm{C}_{\mathrm{n}}{ }^{2}=5^{*} 10^{-14}$ and path length $=120$ meters. 


\section{CONCLUSION}

In conclusion, a practical method for creating turbulence like phase screens have been established in desired turbulence regimes. The new method for establishing the right measures of addition to the propagated beam has been constructed by arranging the influence of lower and faster fluctuations. It has been found that faster fluctuations are more effective on the strong turbulent regimes. Desired turbulence strength has been established with simulation by suggesting temporal fluctuations in the induced turbulence power in strong turbulence conditions.

\section{ACKNOWLEDGEMENTS}

We would like to acknowledge the support of Bogazici University Research Foundation (BAP Project Numbers: 07A204D and 07S102) and TUBITAK (Project Number: 108E205).

\section{REFERENCES}

[1] Z. S. Wu, H. Y. Wei, R. K. Yang and L. X. Gou., "Study on Scintillation Considering inner and outer scales for laser beam propagation on the slant path through the atmospheric turbulence," Progress In Electromagnetics Research 80, 277-293 (2008).

[2] Schmidt, J. D. "Numerical Simulation of Optical Wave Propagation with examples in Matlab," SPIE Press, (2010).

[3] Martin J. M. and Flatte S. M., "Simulation of point-source scintillation through three-dimensional random media," J. Opt. Soc. Am. A 7(5), pp. 838-847 (1990).

[4] Flatte S. M., Wang G. Y., and Martin J., "Irradiance variance of optical waves through atmospheric turbulence by numerical simulation and comparison with experiment," J. Opt. Soc. Am. A 10(11), pp. 2363-2370 (1993).

[5] Sjoqvist, L., Henriksson, M. and Steinvall, O. "Simulation of laser beam propagation over land and sea using phase screens- a comparison with experimental data," Proc. Of SPIE 5989, 59890D, (2005).

[6] Martin J.M. and Flatte S. M., "Intensity images and statistics from numerical simulation of wave propagation in 3-D random media,” APPLIED OPTICS 2711, 2111-2125 (1988). 